Pacific Journal of Mathematics

THE EXISTENCE OF NATURAL FIELD STRUCTURES FO
FINITE DIMENSIONAL VECTOR SPACES OVER LOCAL

Mitchell Herbert TAibleson 


\title{
THE EXISTENCE OF NATURAL FIELD STRUCTURES FOR FINITE DIMENSIONAL VECTOR SPACES OVER LOCAL FIELDS
}

\author{
MitChell H. TAIBleson
}

\begin{abstract}
Let $K$ be a local field (e.g., a $p$-adic or $p$-series field) and $n$ a positive integer. Let $K^{\prime}$ be the unique (up to isomorphism) unramified extension of $K$. It is shown that the natural (modular) norm of $K^{\prime}$ is the $n$th power of the usual $\left(l^{\infty}\right)$ vector space norm of $K^{\prime}$ when $K^{\prime}$ is viewed as an $n$ dimensional vector space over $K$. Further, the two distinct descriptions of the dual of $K^{\prime}$ (which is isomorphic to $K^{\prime}$ ) that arise from the field model and vector space model are isomorphic under a $K$-linear isomorphism of $K^{\prime}$ as a vector space over $K$, and the isomorphism is norm preserving.
\end{abstract}

1. If $\boldsymbol{R}^{n}$ is $n$-dimensional Euclidean space and $n>1$, then the only case for which $\boldsymbol{R}^{n}$ has a (commutative) field structure is $n=2$. In that case $\boldsymbol{R}^{2}$ can be identified as the additive group of $C$, the complex numbers, and the norms for $\boldsymbol{R}^{2}$ and $\boldsymbol{C}$ are compatible in the following sense: Let $(x, y) \in \boldsymbol{R}^{2}$ and consider the correspondence $(x, y) \leftrightarrow z=x+i y$. The norm of $(x, y) \in \boldsymbol{R}^{2}$ is $|z|_{\boldsymbol{R}^{2}}=|(x, y)|_{\boldsymbol{R}^{2}}=$ $\left(x^{2}+y^{2}\right)^{1 / 2}$. Let $d z$ be Haar measure on $C$. We define $N_{c}(\mathrm{w})=w \bar{w}$ and $\bmod _{c}(w)$ by the relation $d(w z)=\bmod _{c}(w) d z$. We obtain, as is well known: $|z|_{\boldsymbol{R}^{2}}^{2}=N_{C}(z)=\bmod _{C}(z)$.

We will show that if $K$ is a local field (e.g., if $K$ is a $p$-adic field) and $n$ is an integer greater than 1 , then $K^{n}$, the $n$-dimensional vector space over $K$, has a field structure, as a local field, which is compatible with the usual vector space norm of $K^{n}$, in the same sense as above.

The reader is referred to [3; Ch. I] for a review of the basic facts about local fields and to [4; Chs. I-II] for many details and proofs.

2. Let $K$ be a local field; which is to say a locally compact, nondiscrete field that is not connected. The $K$ is totally disconnected. Such a field is either a $p$-adic field, a finite algebraic extension of a $p$-adic field or the field of formal Laurent series over a finite field. The ring of integers, $\mathfrak{O}$, in $K$ is the unique maximal compact subring of $K$. The prime ideal, $\mathfrak{D}$, in $\mathfrak{D}$, is a maximal ideal that is principal, $\mathfrak{D} / \mathfrak{P} \cong G F(q)$, a finite field. There is a norm on $K$, $|\cdot|_{K}: K^{*} \rightarrow[0, \infty)$, such that $|x+y|_{K} \leqq \max \left[|x|_{K},|y|_{K}\right]$. (This is known as the ultrametric inequality.) $\quad \mathfrak{O}=\left\{|x|_{K} \leqq 1\right\} . \quad \mathfrak{P}=\left\{|x|_{K}<1\right\}$. 
The group of units, $\mathfrak{D}^{*}$, in $K^{*}$ (the multiplicative group of $K$ ) is $\left\{|x|_{K}=1\right\}$. The norm, $|\cdot|_{K}$, arises naturally since $|y|_{K}=\bmod _{K}(y)$ where $\bmod _{K}(y)$ is the module of the endomorphism $x \rightarrow x y$; that is, $\bmod _{K}(0)=0$ and if $y \neq 0$ then $d(y x)=\bmod _{K}(y) d x$, where $d x$ is Haar measure on $K^{+}$, the additive group of $K$. The $n$-dimensional vector space over $K, K^{n}$, is endowed with a norm as follows: $x=$ $\left(x_{1}, \cdots, x_{n}\right) \in K^{n},|x|_{K^{n}}=\max _{k}\left|x_{k}\right|_{K}$. As Weil points out [4, Ch. II $\S 1$, this norm is "natural" in the sense that any $K$-homogeneous, ultrametric norm on $K^{n}$ gives rise to the same topology on $K^{n}$ as $|\cdot|_{K^{n}}$.

Let $n$ be a positive integer, $n \geqq 2$. If $x \in K^{*}$ then $|x|_{K}=q^{k}$ for some $k \in \boldsymbol{Z}$. Furthermore, the principal ideal $\mathfrak{P}$ is generated by $\mathfrak{p}=\mathfrak{B},|\mathfrak{p}|_{K}=q^{-1}$. The polynomial $x^{n}-\mathfrak{p}$ is clearly irreducible over $K$ since if $x$ is a root $|x|_{K}=q^{-1 / n}$, which is impossible. Thus, there is is an algebraic field extensions of $K$ of degree $n$ for all $n$.

Let $K[\tau]$ be a given finite algebraic field extension of $K$ of degree $n . K[\tau]$ is a local field and is endowed with an (analytically) natural norm, $\bmod _{K[\tau]}(\cdot)$. We note that if $y \in K$ then $\bmod _{K[\tau]}(y)=$ $|y|_{K}^{n}$ [4; p. 6]. If $K[\tau]$ is normal over $K$ then $K[\tau]$ is also endowed with an (algebraically) natural norm as follows: Let $A$ be the automorphism group of $K[\tau]$ over $K$. Then one defines the norm function $N(y)=\prod_{\alpha \in A} \alpha(y)$. $\quad N(y) \in K$ for all $y \in K[\tau]$ and the norm is defined by $x \rightarrow|N(x)|_{K}$. Clearly, if $x \in K,|N(x)|_{K}=|x|_{K}^{n}$. In fact, as is well known, $|N(x)|_{K}=\bmod _{K[\tau]}(x)$ for all $x \in K[\tau]$. This follows easily from the observation that if $x \in K[\tau]$ and $\alpha \in A, \bmod _{K[\tau]}(\alpha(x))=$ $\bmod _{K[\tau]}(x)$ since automorphisms of local fields have module 1 [4; p. 14].

$$
\begin{aligned}
|N(x)|_{K} & =\left\{\bmod _{K[\tau]}(N(x))\right\}^{1 / n} \\
& =\left\{\bmod _{K[\tau]}\left(\prod_{\alpha \in A} \alpha(x)\right)\right\}^{1 / n} \\
& =\left\{\prod_{\alpha \in A} \bmod _{K[\tau]}(\alpha(x))\right\}^{1 / n} \\
& =\left\{\bmod _{K[\tau]}(x)\right\}^{n \cdot 1 / n}=\bmod _{K[\tau]}(x) .
\end{aligned}
$$

If $x \in K[\tau], x=x_{1}+x_{2} \tau+\cdots+x_{n} \tau^{n-1}, x_{k} \in K$. The correspondence $x_{1}+\cdots+x_{n} \tau^{n-1} \leftrightarrow\left(x_{1}, \cdots, x_{n}\right)$ is a linear isomorphism of $K[\tau]$ and $K^{n}$ as vector spaces over $K$. Using that isomorphism we will denote each element in the corresponding pair with the single symbol $x$. It would be nice to find an extension $K[\tau]$ of degree $n$ such that $\bmod _{K[\tau]}(x)=|x|_{K^{n}}^{n}=\max _{k}\left|x_{k}\right|_{K}^{n}$. (Note that this holds for all $x \in K$.)

We can do this with the aid of Corollaries 2-3 in Chapter III $\S 4$ of Weil's book, Basic Number Theory [4]. According to these results, if $K$ is a local field, $n \geqq 2$ is an integer and $\mathfrak{D} / \mathfrak{F} \cong G F(q)$ where $q$ is a power of a prime $p$, then there is a field $K^{\prime}$ which 
is the unique (up to isomorphism) unramified extension of $K$ of degree $n$, and $K^{\prime}$ is a cyclic Galois extension of $K, K^{\prime}=K[\tau]$ where $\tau$ is a root of unity (of order prime to $p$ ).

We denote $\mathfrak{Q}, \mathfrak{\Omega}^{\prime}$ the rings of integers of $K$ and $K^{\prime} ; \mathfrak{P}, \mathfrak{P}^{\prime}$ the prime ideals of $\mathfrak{Q}$ and $\mathfrak{Q}^{\prime}$ and we let $\mathfrak{t}=\mathfrak{Q} / \mathfrak{P}, \mathfrak{k}^{\prime}=\mathfrak{Q}^{\prime} / \mathfrak{P}^{\prime}$. From the two corollaries we obtain that $\mathfrak{f}^{\prime}=\mathfrak{H}\left[\rho^{\prime}(\tau)\right]$ where $\rho^{\prime}$ is the canonical homomorphism of $K^{\prime}$ onto $\mathfrak{f}^{\prime}$ and that $\mathfrak{f}^{\prime}$ is an extension of $\mathfrak{t}^{\mathfrak{t}}$ of degree $n$.

THeOREm. Let $K^{\prime}=K[\tau]$ be the unramified extension of $K$ of degree n. Then $|N(x)|_{K}=\bmod _{K^{\prime}}(x)=|x|_{K^{n}}^{n}$ for all $x \in K^{\prime}$.

It has been suggested that this theorem is well-known to experts. However, no one has yet been able to give a reference for the second of the two equalities. Since this is needed for the applications in $\S 3 \mathrm{I}$ will sketch a proof.

Proof. Since $K^{\prime}$ is normal over $K$ we only need to show the second equality; namely,

$$
\bmod _{K^{\prime}}\left(x_{1}+x_{2} \tau+\cdots+x_{n} \tau^{n-1}\right)=\max _{k}\left[\bmod _{K}\left(x_{k}\right)\right]^{n} .
$$

(a) $\forall x \in K, \bmod _{K^{\prime}}(x)=\left[\bmod _{K}(x)\right]^{n}$. See $[4 ;$ p. 6]

(b) $\bmod _{K^{\prime}}(\tau)=1$. Note that $\tau$ is a root of unity.

(c) $\bmod _{K^{\prime}}(x) \leqq \max _{k}\left[\bmod _{K}\left(x_{k}\right)\right]^{n}$. Use the fact that $\bmod _{K^{\prime}}(\cdot)$ is ultrametric and apply (a) and (b).

(d) We may assume, without loss of generality, that $\max _{k}\left[\bmod _{K}\left(x_{k}\right)\right]=1$ and that at least two coefficients $x_{k}, x_{l}, k \neq l$ are such that $\bmod _{K}\left(x_{k}\right)=\bmod _{K}\left(x_{l}\right)=1$.

The reduction to $\max _{k}\left[\bmod _{k}\left(x_{k}\right)\right]=1$ is by homogeneity. If there is only one coefficient $x_{k}(\operatorname{say} k=1)$ such that $\bmod _{K}\left(x_{k}\right)=1$ then the result follows from the ultrametric inequality. For suppose $\bmod _{K}\left(x_{1}\right)=$ 1 and $\bmod _{K}\left(x_{k}\right)<1, k \neq 1$. Then from (c) $\bmod _{K^{\prime}}\left(x_{2} \tau+\cdots+x_{n} \tau^{n-1}\right)<$ 1 and from (a) $\bmod _{K^{\prime}}\left(x_{1}\right)=1$. An easy consequence of the ultrametric inequality is that if $\left|y_{1}\right| \neq\left|y_{2}\right|$ then $\bmod _{K^{\prime}}\left(y_{1}+y_{2}\right)=$ $\max \left[\bmod _{K^{\prime}}\left(y_{1}\right), \bmod _{K^{\prime}}\left(y_{2}\right)\right]$. Thus $\bmod _{K^{\prime}}(x)=\bmod _{K^{\prime}}\left(x_{1}\right)=1$.

Hence our result is proved if we show, under the assumptions of (d) that $\bmod _{K^{\prime}}(x)<1$ will lead to a contradiction.

(e) $\bmod _{K^{\prime}}(x)<1$ iff $\rho^{\prime}(x)=0$. Use the characterization: $\mathfrak{P}^{\prime}=$ $\left\{x: \bmod _{K^{\prime}}(x)<1\right\}$.

(f) $\rho^{\prime}(x)$ is a polynomial in $\rho^{\prime}(\tau)$ with coefficients in $\mathfrak{f}$, it is of degree less than $n$ and has at least two nonzero coefficients. This follows from (d) and the remarks preceding the theorem.

(g) The desired contradiction follows from (e) and (f). If $\bmod _{K^{\prime}}(x)<1$ then $\rho^{\prime}(\tau)$ is the root of a monic polynomial over $\mathfrak{l}^{\prime}$ of 
degree less than $n$. This implies that $\left[\mathfrak{t}^{\prime}: \mathfrak{t}^{\prime}\right]<n$, but $\left[\mathfrak{f}^{\prime}: \mathfrak{t}^{\prime}\right]=n$. Hence $\bmod _{K^{\prime}}(x)=1$, which proves the theorem.

3. We now give a few simple consequences of the theorem in $\S 2$.

Throughout this section $K$ is a fixed local field with norm: $|x|_{K}=\bmod _{K}(x), n$ is an integer greater than $1, K^{\prime}=K[\tau]$ is the unramified extension of $K$ of degree $n$ with norm: $|x|_{K^{\prime}}=\bmod _{K^{\prime}}(x)$, $K^{n}$ is the $n$-dimensional vector space over $K$ with norm $|x|_{K^{n}}=$ $\max _{k}\left|x_{k}\right|_{K}, x=\left(x_{1}, \cdots, x_{n}\right), x_{k} \in K$. As in $\S 2$ if $x \in K^{\prime}=K[\tau]$ we have $x=x_{1}+\cdots+x_{n} \tau^{n-1}$ and we identify

$$
\left(x \in K^{\prime}\right) \longleftrightarrow\left(x=\left(x_{1}, \cdots, x_{n}\right) \in K^{n}\right) \text { so that }|x|_{K^{\prime}}=|x|_{K^{n}}^{n} .
$$

We recall that if $\mathfrak{Q}$ is the ring of integers in $K$, and $\mathfrak{P}$ is the prime ideal in $\mathfrak{Q}$ then $\mathfrak{Q} / \mathfrak{P} \cong G F(q)$, a finite field. We also have the fractional ideals $\mathfrak{S}^{k}=\left\{|x|_{K} \leqq q^{-k}\right\}, k \in \boldsymbol{Z}$.

In $K^{\prime}$ we proceed in the same way. Let $R$ be the ring of integers in $K^{\prime}, P$ the prime ideal in $R$ so $R / P \cong G F\left(q^{n}\right)$. The fractional ideals are $P^{k}=\left\{|x|_{K^{\prime}} \leqq\left(q^{n}\right)^{-k}\right\}$. We note that $R=P^{0}$, $P=P^{1}$. Details may be found in [3; Ch. I $\left.\S 5\right]$.

For the vector space $K^{n}$ one defines a neigeborhood system at 0 , with the collection of balls with centers at the origin. Namely, we set $P_{1}^{k}=\left\{|x|_{K^{n}} \leqq q^{-k}\right\}$ and then let $R_{1}=P_{1}^{0}$ and $P_{1}=P_{1}^{1}$. From the fact that $|x|_{K^{\prime}}=|x|_{K^{n}}^{n}$ it follows that $P_{1}^{k}=P^{k}$ for all $k \in Z$ and hence $R_{1}=R, P_{1}=P$. Consequently we drop the subscripts. See [3; ch. III $\S 1]$ for details of this construction for $K^{n}$.

As additive groups (and as $n$-dimensional vector spaces over $K), K^{\prime}$ and $K^{n}$ agree so additive harmonic analysis, Haar measure, etc., all agree on these two different models for $K^{n}$. We now examine the two different descriptions of the dual of $K^{n}$ that arise from the two models.

We fix a character on $K^{+}$that is trivial on $\mathfrak{D}$, but is nontrivial on $\mathfrak{P}^{-1}$. This character is denoted $\chi$. (See [3; Ch. I §5] for details.) The dual of $K^{n}$ is put into a linear isomorphism with $K^{n}$, as a vector space over $K$, by the identification $y \leftrightarrow \chi_{y}^{1}, \chi_{y}^{1}(x)=\chi(x \cdot y)=$ $\chi\left(x_{1} y_{1}+\cdots+x_{n} y_{n}\right)$.

The dual of $K^{\prime}$ (as an additive group) is put into a linear isomorphism with the additive group of $K^{\prime}$ as follows: One first defines the trace function, $\operatorname{Tr}(x)=\sum_{\alpha \in A} \alpha(x)$, where $A$ is the automorphism group of $K^{\prime}$ over $K$. It is known that $\operatorname{Tr}$ maps $K^{\prime}$ onto $K\left[4 ;\right.$ p. 139] and since $K^{\prime}$ is unramified over $K$ we have that $T r$ maps $P^{k}$ onto $\mathfrak{S}^{k}$ for all $k$ [4; p. 141]. The dual of $K^{\prime}$ is then identified with $K^{\prime}$ by the correspondence $y \leftrightarrow \chi_{y}^{2}, \chi_{y}^{2}(x)=\chi(\operatorname{Tr}(x y))$. 
Thus, given any $y \in K^{\prime}$, there is an $L(y) \in K^{\prime}$ such that $\chi_{y}^{1}=$ $\chi_{L(y)}^{2}$, which is to say

$$
\chi\left(x_{1} y_{1}+\cdots+x_{2} y_{2}\right)=\chi(\operatorname{Tr}(x L(y))) \text { for all } x \in K^{\prime},
$$

and the map $y \leftrightarrow L(y)$ is a $K$-linear isomorphism of $K^{\prime}$ (or, more properly, of the dual of the additive group of $K^{\prime}$ ). Moreover, this linear map preserves the norm of $y$; that is, $|L(\mathrm{y})|_{K^{\prime}}=|y|_{K^{\prime}}$ for all $y \in K^{\prime}$.

We first note that $\chi_{y}^{1} \equiv 1$ iff $y=0$ and if $|y|_{K^{\prime}}=q^{k n}$, then $\chi_{y}^{1}$ is trivial on $P^{k}$ but is nontrivial on $P^{k-1}$. (See [3; Ch. III §1].) From the fact that $\operatorname{Tr}$ maps $P^{k}$ onto $\mathfrak{P}^{k}$ and the fact that $\chi$ is trivial on $\mathfrak{O}$ but is nontrivial on $\mathfrak{P}^{-1}$ we see that $\chi_{L(y)}^{2} \equiv 1$ iff $L(y)=0$ and that if $|L(y)|_{K^{\prime}}=q^{l n}$, then $\chi_{L(y)}^{2}$ is trivial on $P^{l}$ but is nontrivial on $P^{l-1}$. Thus, $|L(y)|_{K^{\prime}}=|y|_{K^{\prime}}$.

Therefore, these two representations of the dual of $K^{\prime}$ as an additive group have the same induced norm and hence the same induced metric.

Note also that the prime ideal $P$ is generated by any element $p \in P$ such that $|p|_{K^{\prime}}=q^{-n}$. $\mathfrak{P}$ is generated by $\mathfrak{p} \in \mathfrak{P}$, where $|\mathfrak{p}|_{K}=$ $q^{-1}$. But $\mathfrak{p} \in P$ and $|\mathfrak{p}|_{K^{\prime}}=|\mathfrak{p}|_{K}^{n}=q^{-n}$, so $P$ is generated in $R$ by the same element, $\mathfrak{p}$, that generates $\mathfrak{P}$ in $\mathfrak{D}$.

These last few results are simply the working out of notational consequences of the identity $|x|_{K^{n}}^{n}=|x|_{K^{\prime}}$.

When we study Calderón-Zygmund kernels on $K$ we look at functions of the form $\Omega(x) /|x|_{K}$ where $\Omega(x)$ is homogeneous of degree 0 in the sense that $\Omega\left(\mathfrak{p}^{k} x\right)=\Omega(x), \forall x \in K, k \in Z$ [3; Ch. VI $\S 4$ ]. Thus, on $K^{\prime}$ we examine functions of the form $\Omega(x) /|x|_{K^{\prime}}$ where $\Omega$ is homogeneous of degree 0 in the sense that $\Omega\left(\mathfrak{p}^{k} x\right)=\Omega(x)$ for all $x \in K^{\prime}, k \in \boldsymbol{Z}$ and " $\mathfrak{p}^{k} x$ " is multiplication of $x \in K^{\prime}$ by $\mathfrak{p}^{k} \in K^{\prime}$.

When we examine such kernels on $K^{n}$, the functions are of the form $\Omega(x) /|x|_{K^{n}}^{n}$ where $\Omega$ is homogeneous of degree zero in the sense that $\Omega\left(\mathfrak{p}^{k} x\right)=\Omega(x)$ for all $x \in K^{n}, k \in Z$ and " $\mathfrak{p}^{k} x$ " is scalar multiplication of $x \in K^{n}$ by $\mathfrak{p}^{k} \in K$. But these two "multiplications" agree and since $|x|_{K^{n}}^{n}=|x|_{K^{\prime}}$ the classes of kernels that would arise from these two approaches to $K^{n}$ are the same class.

We will continue the analysis of these kernels a little further. Note that $R^{*}=\left\{|x|_{K^{\prime}}=1\right\}$ is a multiplicative group. It is the group of units in $\left(K^{\prime}\right)^{*}$. We consider (as in [2] and [3; Ch. II §4]) the collection $\left\{\pi_{k l}\right\}_{k=0, l=0}^{\infty} l^{l}$ of unitary multiplicative characters on $R^{*}$, where $\pi_{k l}$ is ramified of degree $k$ and $l_{k}=q^{k n}\left(1-q^{-n}\right)^{2}, k \geqq 2$, $l_{0}=1, l_{1}=q^{n}-2 . \quad\left\{\left(1-q^{-n}\right) \pi_{k l}\right\}$ is a complete orthonormal system on $R^{*}$ and $\pi_{k l}$ is the local field analogue of a spherical harmonic of degree $k$. 
Consider $\Omega(x) /|x|_{K^{n}}^{n}$ as above with $\int_{R^{*}} \Omega(x) d x=0$. Then $\Omega$ can be considered as a function on $R^{*}$ and we may write, formally,

$$
\begin{aligned}
& \Omega(x) \sim \sum_{k=1}^{\infty} \sum_{l=1}^{l_{k}} c_{k l} \pi_{k l}(x) \text { and so } \\
& \qquad(x) /|x|_{K^{n}}^{n} \sim \sum \sum c_{k l} \pi_{k l}(x) /|x|_{K^{\prime}} .
\end{aligned}
$$

The Fourier transform of the principal value distribution induced by $\Omega(x) /|x|_{K^{\prime}}$ is a function which is homogeneous of degree zero. Call that function $\hat{\Omega}$. Using the results for the gamma function [3; Ch. II §5] it is easy to see that $\hat{\Omega}(x) \sim \sum \sum c_{k l} \Gamma\left(\pi_{k l}\right) \pi_{k l}^{-1}(x)$. That is, the map $\Omega \rightarrow \hat{\Omega}$ is essentially, a multiplier transform on the group $R^{*}$ and the behaviour of the operator depends on the properties of the distribution $M(x) \sim \sum_{k>1} \Gamma\left(\pi_{k l}^{-1}\right) \pi_{k l}(x)$.

If convolution by the principal value distribution induced by $\Omega(x) /|x|_{K^{\prime}}$ is a bounded operator on any $L^{p}$ space, then it is bounded on $L^{2}$ and this implies that $\hat{\Omega}$ is bounded. What conditions on $\Omega$ imply that $\hat{\Omega}$ is bounded? By the usual arguments for multipliers we see that $\hat{\Omega}$ is bounded whenever $\Omega \in L^{2}\left(R^{*}\right)$ implies that $M \in L^{2}\left(R^{*}\right)$. But $\left|\Gamma\left(\pi_{k l}\right)\right|=q^{-k n / 2}$ [3; Ch. II $\left.\S 5\right]$ and since $l_{k}=q^{k n}\left(1-q^{-n}\right)^{-2}$, $k \geqq 2$, we see that $M \notin L^{2}\left(R^{*}\right)$. (See [2] for details and extensions.)

Similarly $\hat{\Omega}$ is bounded whenever $\Omega \in L^{\infty}\left(R^{*}\right)$ implies that $M$ is a finite Borel measure. When $q$ is odd, a careful examination shows that $M$ is not a finite Borel measure and thus the singular integral operator $f \rightarrow f *\left(\mathrm{P} . \mathrm{V} . \Omega(x) /|x|_{K^{\prime}}\right)$ is not necessarily bounded on $L^{2}\left(K^{\prime}\right)$ when $\Omega \in L^{\infty}\left(R^{*}\right)$. The same result also follows for $\Omega$ continuous on $R^{*}$. (This is the essential part of Daley's argument in [1].)

As a final example, we state an especially simple $F$. and $M$. Riesz theorem for $K^{n}$. Let $q$ be odd, $\mathfrak{O} / \mathfrak{P} \cong G F(q)$ and $n$ be any positive integer. Then there is a singular integral operator of the Calderón-Zygmund type, $f \rightarrow \widetilde{f}=f^{*}\left(P . V . \Omega(x) /|x|_{K}^{n}\right)$ with the following property. If $\mu$ is a finite Borel measure and $\tilde{\mu}$ is a finite Borel measure, then $\mu$ is absolutely continuous. Viewed from the perspective of $K^{\prime}$ we choose $\Omega(x)=\pi(x)$ where $\pi$ is any unitary character on $R^{*}, \pi$ ramified of degree 1 , homogeneous of degree 0 and odd. This was shown by Chao for $n=1$ [3; Ch. VII $\S 3]$.

\section{REFERENCES}

1. J. Daley, The invalidity of the Calderón-Zygmund inequality for singular integrals over local fields, Bull. Amer. Math. Soc., 18 (1975), 896-899.

2. W-N. Liu, On singular integral kernels over local fields, Dissertation, Washington University, 1974.

3. M. H. Taibleson, Fourier Analysis on Local Fields, Math. Notes No. 15, Princeton 
Univ. Press, Princeton, 1975.

4. A. Weil, Basic Number Theory, Springer Verlag, New York, 1967.

Received August 7, 1975. Research supported in part by NSF Grant No. MPS-02411.

WASHINGTON UNIVERSITY 



\title{
PACIFIC JOURNAL OF MATHEMATICS
}

\author{
EDITORS
}

\author{
RICHARD ARENS (Managing Editor) \\ University of California \\ Los Angeles, California 90024
}

\author{
R. A. Beaumont \\ University of Washington \\ Seattle, Washington 98105
}

J. DugundjI

Department of Mathematics

University of Southern California

Los Angeles, California 90007

D. Gilbarg and J. Milgram

Stanford University

Stanford, California 94305

\section{ASSOCIATE EDITORS}
E. F. BECKENBACH
B. H. NEUMANN
F. WOLF
K. Yoshida

\section{SUPPORTING INSTITUTIONS}

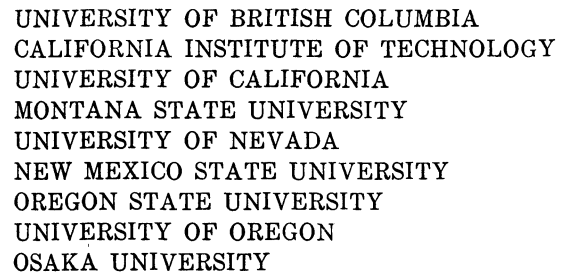

UNIVERSITY OF BRITISH COLUMBIA CALIFORNIA INSTITUTE OF TECHNOLOGY UNIVERSITY OF CALIFORNIA MONTANA STATE UNIVERSITY UNIVERSITY OF NEVADA NEW MEXICO STATE UNIVERSITY OREGON STATE UNIVERSITY UNIVERSITY OF OREGON OSAKA UNIVERSITY

\author{
UNIVERSITY OF SOUTHERN CALIFORNIA \\ STANFORD UNIVERSITY \\ UNIVERSITY OF HAWAII \\ UNIVERSITY OF TOKYO \\ UNIVERSITY OF UTAH \\ WASHINGTON STATE UNIVERSITY \\ UNIVERSITY OF WASHINGTON

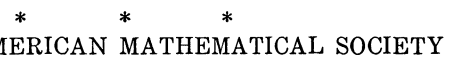

The Supporting Institutions listed above contribute to the cost of publication of this Journal, but they are not owners or publishers and have no responsibility for its content or policies.

Mathematical papers intended for publication in the Pacific Journal of Mathematics should be in typed form or offset-reproduced, (not dittoed), double spaced with large margins. Please do not use built up fractions in the text of your manuscript. You may however, use them in the displayed equations. Underline Greek letters in red, German in green, and script in blue. The first paragraph or two must be capable of being used separately as a synopsis of the entire paper. Items of the bibliography should not be cited there unless absolutely necessary, in which case they must be identified by author and Journal, rather than by item number. Manuscripts, in triplicate, may be sent to any one of the editors. Please classify according to the scheme of Math. Reviews, Index to Vol. 39. All other communications should be addressed to the managing editor, or Elaine Barth, University of California, Los Angeles, California, 90024.

The Pacific Journal of Mathematics expects the author's institution to pay page charges, and reserves the right to delay publication for nonpayment of charges in case of financial emergency.

100 reprints are provided free for each article, only if page charges have been substantially paid. Additional copies may be obtained at cost in multiples of 50 .

The Pacific Journal of Mathematics is issued monthly as of January 1966. Regular subscription rate: $\$ 72.00$ a year (6 Vols., 12 issues). Special rate: $\$ 36.00$ a year to individual members of supporting institutions.

Subscriptions, orders for back numbers, and changes of address should be sent to Pacific Journal of Mathematics, 103 Highland Boulevard, Berkeley, California, 94708.

PUBLISHED BY PACIFIC JOURNAL OF MATHEMATICS, A NON-PROFIT CORPORATION

Printed at Kokusai Bunken Insatsusha (International Academic Printing Co., Ltd.), 8-8, 3-chome, Takadanobaba, Shinjuku-ku, Tokyo 160, Japan.

Copyright (C) 1975 by Pacific Journal of Mathematics Manufactured and first issued in Japan 


\section{Pacific Journal of Mathematics}

\section{Vol. 63, No. 2 \\ April, 1976}

Joseph Anthony Ball and Arthur R. Lubin, On a class of contractive perturbations

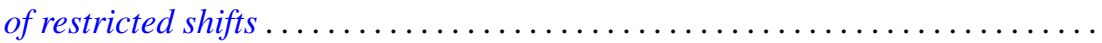

Joseph Becker and William C. Brown, On extending higher derivations generated

by cup products to the integral closure .......................

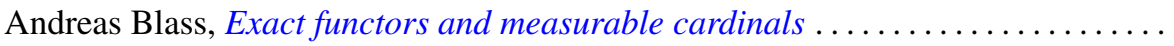

Joseph Eugene Collison, A variance property for arithmetic functions . . . . . . . . . .

Craig McCormack Cordes, Quadratic forms over nonformally real fields with a

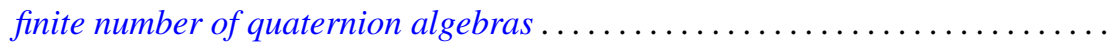

Freddy Delbaen, Weakly compact sets in $H^{1} \ldots \ldots \ldots \ldots \ldots \ldots \ldots \ldots \ldots$

G. D. Dikshit, Absolute Nörlund summability factors for Fourier series ..........

Edward Richard Fadell, Nielsen numbers as a homotopy type invariant. . ........

Josip Globevnik, Analytic extensions of vector-valued functions . . . . . . . . . . . .

Robert Gold, Genera in normal extensions . . . . . . . . . . . . . . . . . . . 389

Solomon Wolf Golomb, Formulas for the next prime

Robert L. Griess, Jr., The splitting of extensions of $S L(3,3)$ by the vector space

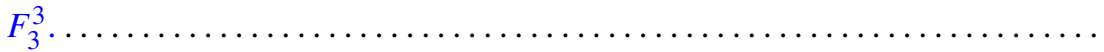

Thomas Alan Keagy, Matrix transformations and absolute summability .........

Kazuo Kishi, Analytic maps of the open unit disk onto a Gleason part.

Kwangil Koh, Jiang Luh and Mohan S. Putcha, On the associativity and commutativity of algebras over commutative rings . ..... . .

James C. Lillo, Asymptotic behavior of solutions of retarded differential difference equations.

John Alan MacBain, Local and global bifurcation from normal eigenvalues ..

Anna Maria Mantero, Sets of uniqueness and multiplicity for $L^{p}$

J. F. McClendon, Embedding metric families

L. Robbiano and Giuseppe Valla, Primary powers of a prime ideal .

Wolfgang Ruess, Generalized inductive limit topologies and barrelledness

properties.

Judith D. Sally, Bounds for numbers of generators of Cohen-Macaulay ideals

Helga Schirmer, Mappings of polyhedra with prescribed fixed points and fixed point indices.

Cho Wei Sit, Quotients of complete multipartite graphs

S. Sznajder and Zbigniew Zielezny, Solvability of convolution equations in $\mathscr{K}_{p}^{\prime}$,

$p>1$.

Mitchell Herbert Taibleson, The existence of natural field structures for finite

dimensional vector spaces over local fields

William Yslas Vélez, A characterization of completely regular fields

P. S. Venkatesan, On right unipotent semigroups ..............

Kenneth S. Williams, A rational octic reciprocity law ............

Robert Ross Wilson, Lattice orderings on the real field .......... 